\title{
Design Technology Research of Aircraft Engine Health Management (EHM) Technologies
}

\author{
Wessam Abousada \\ College of Civil Aviation, Nanjing University of Aeronautics and Astronautics, Nanjing, China \\ Email: wessam@nuaa.edu.cn
}

How to cite this paper: Abousada, W. (2021) Design Technology Research of Aircraft Engine Health Management (EHM) Technologies. Advances in Aerospace Science and Technology, 6, 9-23.

https://doi.org/10.4236/aast.2021.61002

Received: January 12, 2021

Accepted: February 23, 2021

Published: February 26, 2021

Copyright (C) 2021 by author(s) and Scientific Research Publishing Inc. This work is licensed under the Creative Commons Attribution International License (CC BY 4.0).

http://creativecommons.org/licenses/by/4.0/

\section{(c) (i) Open Access}

\begin{abstract}
Aircraft engine is an important guarantee for aircraft safety, and its failure mode and health management have become the top priority. However, there are very few researches on aircraft engine health management. This article mainly summarizes the current research status of aircraft engine health management (EHM) from the aspect of aircraft electronic system, focuses on the overall structure, functional areas and key technologies of EHM system design, points out the design requirements of EHM system, and finally proposes EHM system. The design must improve the monitoring accuracy of the sensor to meet the monitoring requirements of more than $0.1 \%$. High-precision monitoring data is more conducive to engine fault detection and processing, and EHM will therefore develop in the direction of real-time, intelligent, integrated and networked.
\end{abstract}

\section{Keywords}

Aeroengine, Health Management, Prediction, Information Fusion, Life Cycle

\section{Introduction}

Engine Health Management (EHM) [1] [2] is used to ensure aircraft flight safety, reduce usage costs and guarantee costs, take proactive measures to monitor the health of the engine, predict the trend of performance changes, predict the timing of component failures and remaining service life, and take necessary measures to alleviate engine performance degradation, component failure/failure decision-making. According to literature [3], currently, foreign EHM has developed to the engineering verification stage, and many technologies have been verified and demonstrated on platforms such as $\mathrm{C}-17$ and F/A-17, which proves that EHM can significantly improve the engine performance, reduce operating costs and improve engine reliability and safety. China has recognized the im- 
portant value of EHM and has begun to study it, but has not yet formed a specific design scheme and technical approach. Combining the structural characteristics of the EHM system, this article mainly introduces the EHM system design technology, design requirements and development trends.

\section{EHM System}

\subsection{Physical Distribution Structure}

From the perspective of physical distribution, the overall structure of the EHM system is usually composed of two parts: the ground system and the airborne system [2] [4], as shown in Figure 1. The airborne system usually collects data, completes in-flight testing and in-flight surveillance, and then transmits the in-flight data and reports the ground system for further analysis and decision-making. These two systems keep in touch through the communication system of the aircraft to report the engine health status in time, so that the maintenance support system has sufficient preparation time. The airborne subsystem includes signal processing, status monitoring, health assessment, and self-checking and repairing; fault diagnosis, various predictions and forecasting processes require stronger computer processing resources and extensive, complete, and global data and historical files, So it should be configured on the ground subsystem.

\subsection{Information Hierarchy}

The EHM system is a hierarchical and distributed information system, which can be divided into six levels: signal processing layer, condition monitoring layer, health assessment layer, prediction layer, decision support layer and human-computer interaction layer [2] [4], such as shown in Figure 2.

1) Signal processing layer

The signal processing layer processes the data of the engine sensor system and the input signal of the control system, and extracts the characteristic content of

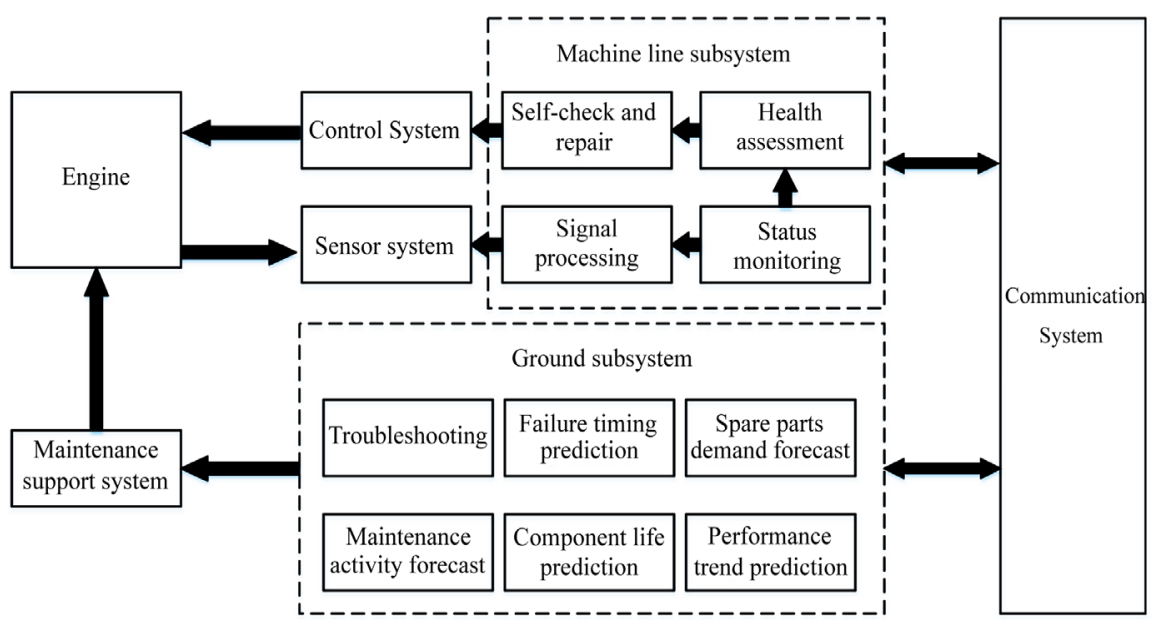

Figure 1. The physical distribution structure of the EHM system. 


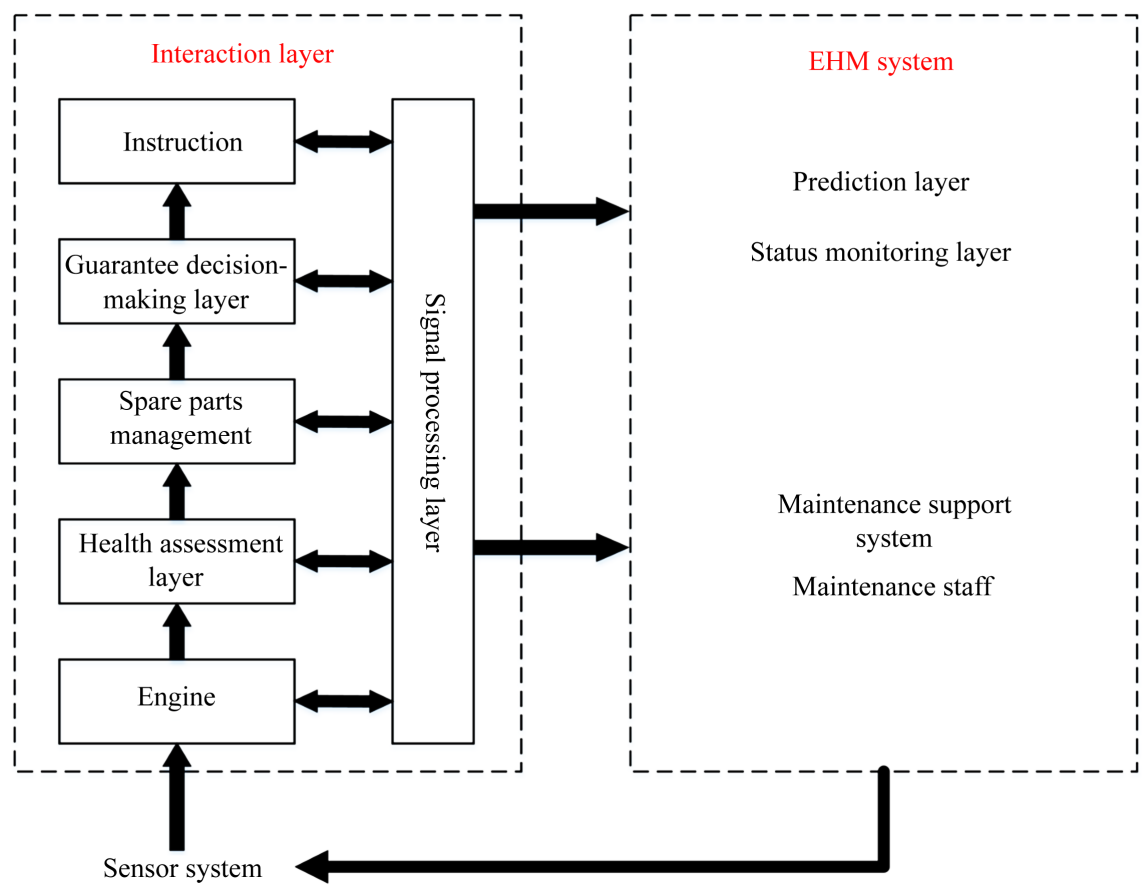

Figure 2. Information hierarchy of EHM system.

the input data according to the given characteristic space. The processing methods usually used in this layer mainly include smoothing filtering, fast Fourier transform, wavelet analysis and other related various statistical methods.

2) State monitoring layer

The state monitoring layer tests and reports on the behavior and structure of engine components, subsystems, materials, and working environment. The main input of this layer includes the output signal of the signal processing layer and the reporting rules of the health assessment layer, and the main output is the status report of the monitored components and subsystems. The main monitoring objects are gas system, fuel system, lubricating oil system and vibration system.

\section{3) Health assessment layer}

The function of the health assessment layer is to integrate information from the status monitoring layer and other health assessment parts, diagnose and report the health status of specified components and subsystems, and perform fault isolation based on this. The node of health assessment should be able to realize diagnosis for testing or health assessment strategy, and command and control system actions to deal with unknown faults or intermittent events. Control actions include control of status monitors and health assessment node reports, as well as control of data collection and task priority. In addition, the health assessment node can also include resource management capabilities to make full use of data storage, information processing and communication resources.

4) Prediction layer 
The prediction layer comprehensively utilizes the information of the previous layers to evaluate and predict the future health status of the engine, indicate the performance trend of the engine, and estimate the remaining service life of key components under different load spectra. And based on this forecast possible spare parts demand and maintenance support activities.

5) Guarantee support layer

This layer processes the data of the status monitoring, health assessment and prediction layers, and generates recommendations for maintenance measures such as replacement and repair. Including implementation and support systems. It has the ability to execute, evaluate and plan, reason about maintenance activities, and manage maintenance resources. At the same time, this layer provides guarantee for maintenance resource management and other levels of processing.

6) Human-computer interaction layer

The human-computer interaction layer is the interface between the EHM system and the engine maintenance support system, which mainly includes the human-machine interface and the machine-machine interface, and has the ability to communicate with all other levels. The man-machine interface is mainly for crew members, ground maintenance personnel and fleet managers; the machine-machine interface is responsible for ensuring the exchange of data and information between the modules of the EHM system and between the EHM system and other external systems.

\section{Main Functional Areas of EHM System}

In order to meet a complex system of aeronautical engine that can involve materials, structure, aerodynamics, thermodynamics, and control, etc., the EHM system has 5 functions [5].

\subsection{Gas Circuit Performance Monitoring}

1) Performance monitoring and component failure diagnosis

When the air flow passes through the engine, the engine failure can be reflected by the aerodynamic thermal parameters and performance parameters of the air circuit. Therefore, the purpose of aerodynamic performance monitoring and component fault diagnosis is to measure the aerodynamic thermal parameters, performance parameters and position parameters of geometrically adjustable components of the engine through sensors to monitor the health of the engine and its gas path components. In the event of a failure, alert the crew in time and analyze the cause of the failure. Therefore, this function is the focus of engine state monitoring and fault diagnosis. Commonly used algorithms include Kalman filter, neural network, genetic algorithm, support vector machine and expert system.

2) Performance trend forecast analysis

As the engine is used, the performance of the engine will inevitably decrease, which requires a predictive assessment of the degradation of its performance. 
Precise engine performance trend prediction is the basis for judging whether the engine is malfunctioning, because this prediction can not only update the engine benchmark performance in the on-board fault diagnosis algorithm, but also improve the accuracy of diagnosis and reduce the occurrence of false alarms. The basis for performance prediction is the engine mathematical model and the historical function and maintenance data of the engine. For complex nonlinear systems such as aeroengines, support vector machines are currently the best method for predicting performance trends [6].

3) Monitoring of gas path debris

Pneumatic debris monitoring is a new technology for monitoring the static electricity carried by engine debris. When the engine is working under normal conditions, the overall static charge in the exhaust gas will remain at a normal level. Therefore, this normal level can be used as a threshold for engine performance degradation. When a surface failure occurs in a gas circuit component, it will be in the exhaust gas. Excessive debris is generated, causing the overall static charge level to exceed the threshold. The type of failure can be judged based on the different characteristics of the change, and an early warning can be given. The gas path debris monitoring can realize real-time monitoring and diagnosis of the surface faults of the engine gas path components. Its greatest advantage is the real-time identification of the faults. It can not only ensure early warning and fault tracking, but also track the development of later faults for maintenance. The arrangement of the plan brings a lot of freedom. This is beyond the reach of other surveillance technologies.

\subsection{Oil Monitoring}

The purpose of lubricating oil monitoring is to use the operating parameters of the lubricating oil system to monitor the physical and chemical properties of the lubricating oil itself and the health of all parts of the engine that are in contact with the lubricating oil, so as to provide information about the health of the engine. Lubricating oil monitoring mainly includes the following three aspects [7] [8].

1) Monitoring of working status of lubricating oil system

The monitoring of the working status of the lubricating oil system is an indication of the pressure, temperature, total volume and consumption of lubricating oil and the parameters of oil filter blockage. The methods include over-limit warning and trend analysis. For example, oil nozzle, oil filter clogging or abnormal pressure regulating work may cause the oil pressure to increase; while leakage, oil pipe rupture, and abnormal operation of the pressure regulating valve will cause the oil pressure to decrease. Too high lubricating oil temperature and other lubricating oil system monitoring parameters can reflect the failure of the engine subsystem. Monitoring the amount of oil and the amount of added oil can get information about excessive oil consumption and oil leakage.

2) Monitoring of oil debris 
Lubricating oil not only has the function of lubrication and cooling, but also has the function of transporting debris. The task of monitoring oil debris is to monitor the health of engine parts that come into contact with lubricating oil, and find out the debris of these parts due to surface failures in time, so as to avoid second damage to the engine. The monitoring also includes two parts: onboard and on the ground: the onboard monitoring uses sensors for real-time analysis to warn of overtime; the ground monitoring part analyzes the debris in the lubricating oil samples collected on the ground.

3) Monitoring of physical and chemical properties of lubricating oil

The physical and chemical performance monitoring of the lubricating oil can provide information on the state of the lubricating oil and abnormal operation of some engines. Ventilation, temperature, consumption of lubricating oil, system capacity and composition, etc. may affect the rate and degree of deterioration of the physical and chemical properties of lubricating oil. The rational properties such as oxidation, additional loss, colloidal impurity content and total acidity of lubricating oil can be monitored to determine the availability of lubricating oil.

\subsection{Vibration Monitoring}

Vibration monitoring focuses on the identification of dangerous vibration conditions at all working speeds, and avoids 2 damages caused by engine component degradation. Vibration monitoring can realize early detection of engine damage, analyze the rate of change and trend of vibration parameters, and find potential failures, thereby ensuring the structural integrity of the engine and improving flight safety.

Vibration monitoring consists of two parts: onboard and underboard. The onboard part compares the vibration amplitude measured by a specific part of the engine with a preset threshold, and sends a warning to the cockpit, monitoring system or maintenance system when the limit is exceeded; the offboard part uses advanced algorithms and models to compare onboard data Do further processing, monitor the vibration trend, and issue an abnormal alarm in advance so that maintenance personnel can take timely measures.

\subsection{Lifetime Monitoring}

Life time monitoring includes two parts: airborne and ground [9]. The airborne part tracks the use or damage of components, and the ground part is responsible for predicting the remaining life of components (such as disks and blades) and making decisions based on this. With the improvement of algorithms and the development of cumulative damage modeling, the current development trend is to use sensor measurement results, and integrate component design models and historical data to accurately predict the remaining life of components. Ensuring the absolute reliability of the remaining life is the main problem currently facing. This requires the development of more effective sensors, the design of more ac- 
curate models and algorithms, and the strengthening of in-depth research on fatigue, oxidation, creep and other damage mechanisms and their interactions.

\subsection{Self-Inspection Repair and Maintenance Support}

Self-checking and repairing refers to the corresponding monitoring and active repairing of various abnormal conditions of the engine performed by the EHM system in the air, minimizing the impact of the engine under various unfavorable conditions, and continuously ensuring flight safety and safe landing of the aircraft. It is based on the evaluation of the impact of various abnormal conditions of the engine, and the assistance of the engine control system is needed in the implementation of specific measures [2] [8]. In order to reduce the cost of engine use and improve the efficiency of maintenance support, EHM must support the maintenance support system. With EHM support, it is possible to keep the engine's health status synchronized with the working status of the maintenance support system, improve the efficiency of each link of the maintenance support system, and realize the intelligentization of spare parts configuration and inventory management and the "intelligent support" of the engine [10]; Managers can update the availability of engines and aircraft in time and make mission decisions.

\section{Key Technologies of EHM System Design}

The EHM system design includes sensors, data management, algorithms and fault diagnosis and prediction technology, integration and communication and other technologies.

\subsection{Sensor Technology}

In the first stage of EHM design, first determine the engine subsystem to be monitored; then select the applicable sensing technology. The EHM system must be able to provide comprehensive engine information and find the best solution for comprehensive multi-sensor data, so that the most state information can be obtained with the least amount of equipment. Therefore, various sensors must be used correctly. Because the optimal control of the engine is achieved by processing the output signal of the sensor to obtain the optimal control result, and then controlling the action of the engine's actuator. The sensor is a key component in the design of the engine health management system. With the development of the engine health management system, the requirements for sensor performance are getting higher and higher, such as integration, multi-function, intelligence, more effective and more accurate.

\subsection{Data Management Technology}

The implementation objects of the EHM system may come from different departments and different types of commercial or military engines. The system needs and generates a large amount of data, the logical structure is complex, the 
demand for diagnosis and prediction data is large, and the real-time and security requirements of the data are high. In order to improve the versatility and efficiency of the EHM system, reduce the cost

Therefore, to realize cross-platform EHM, it is necessary to manage engine data of different platforms in the future. The data management scheme adopted for the characteristics of EHM is shown in Figure 3. Engine data from different platforms is converted into a unified format according to the same conversion rules through data converters, and stored in the form of standard data files and databases for various diagnosis, Prediction algorithms, and visualization and other processing programs are used [11].

\subsection{Algorithms and Fault Diagnosis and Prediction Technology}

\subsubsection{Algorithm}

Algorithms include data-driven algorithms and model-based algorithms. Combining the two algorithms is a hybrid algorithm, which combines the advantages of the two algorithms. There are many specific algorithms, the latest and effective ones are introduced below.

1) Anomaly detection technology

When the engine is in an abnormal state, the software algorithm used to detect the occurrence of the abnormal state is an abnormal detection technology. The methods of judging abnormal conditions include: verifying whether the relationship between the parameters conforms to the basic laws of physics.

Sample data for each time period to train the experimental mode of the engine system or subsystem; use the engine analysis model on board. Anomaly detectors are usually built using neural networks (ANN), and the most commonly used form is radial basis function (RBF) ANN, as shown in Figure 4.

The model is divided into several parts, composed of a series of RBFANN, each RBFANN represents a typical flight envelope or maneuvering characteristics, which improves the accuracy of detection.

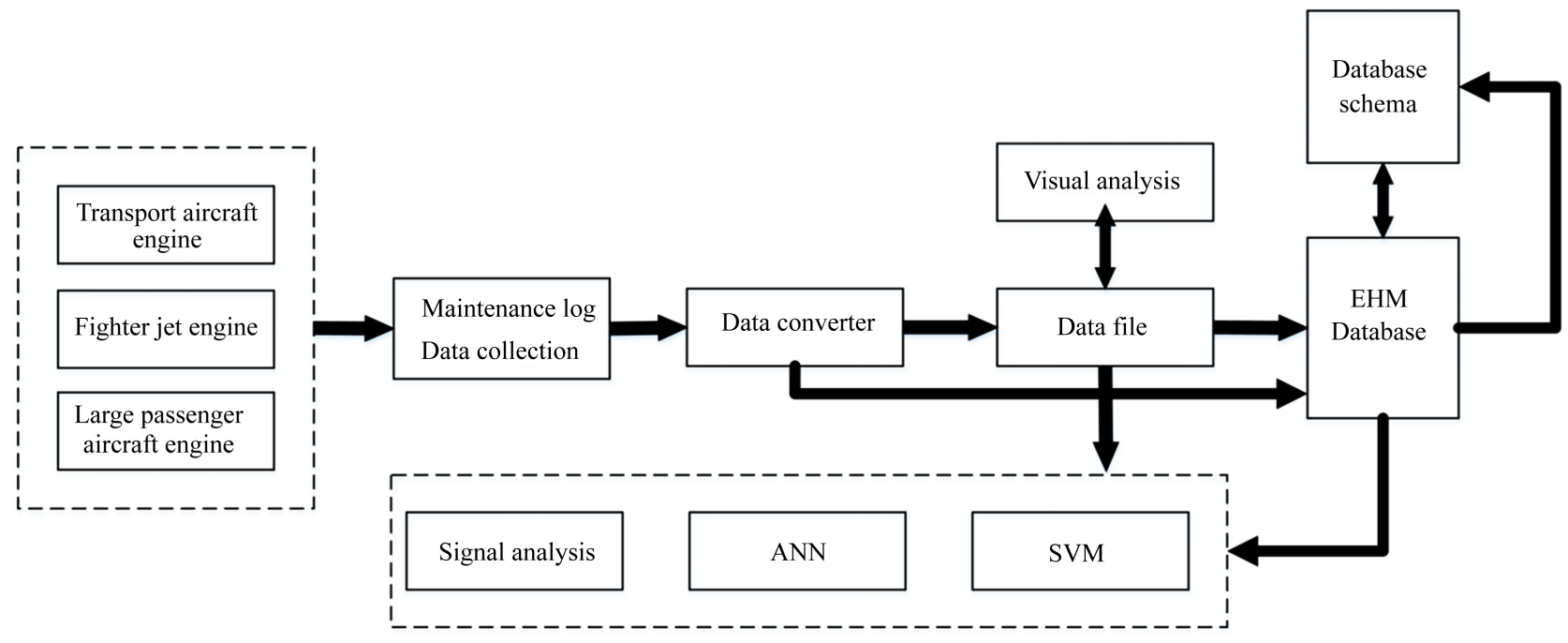

Figure 3. EHM data management scheme. 


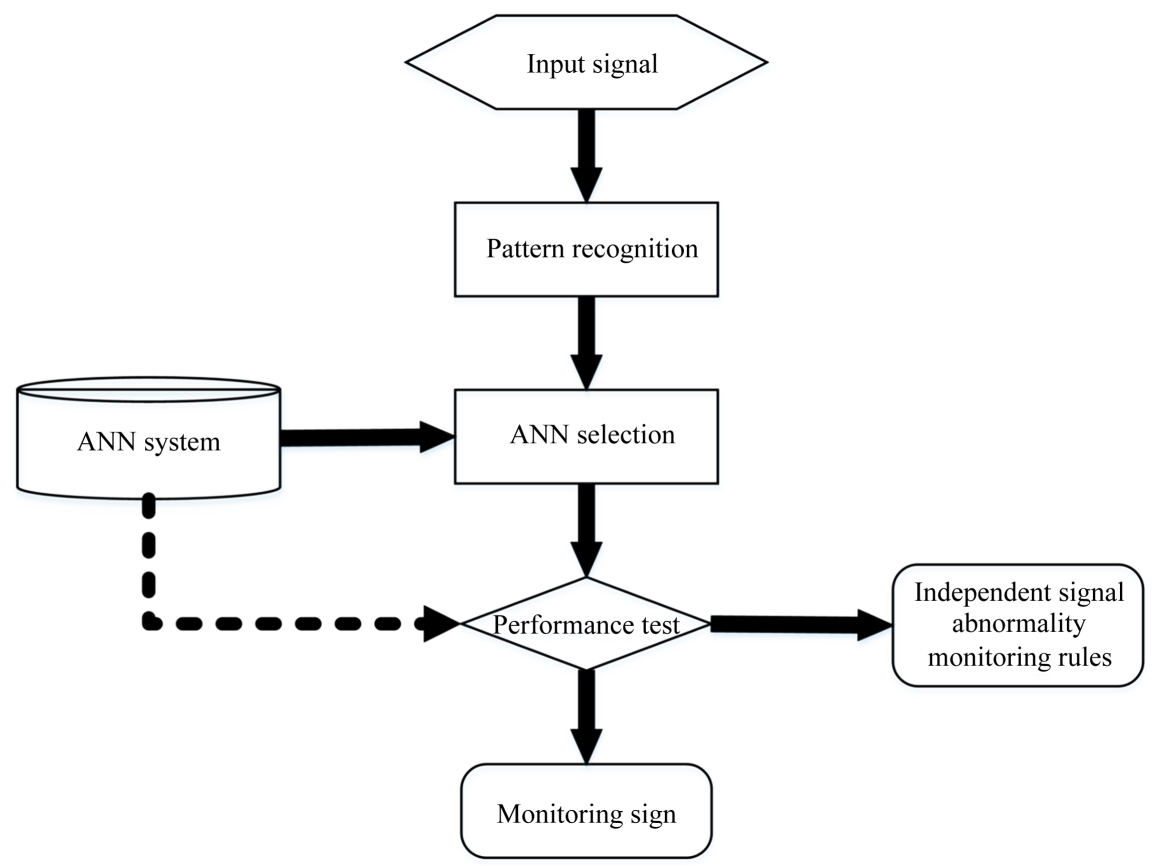

Figure 4. Abnormal diagnosis scheme.

\section{2) Artificial neural network}

There are three basic types of commonly used neural networks: feedforward neural networks, RBF neural networks and autoassociative neural networks. These ANNs are mainly used for parameter relationship modeling and fault detection and analysis. At present, ANN has been widely used in engine health management and is effective in long-term degradation modeling detection and short-term drift.

3) Support vector machine

Support vector machine is a machine learning algorithm proposed by Vapnik in the mid-1990s [11]. The support vector machine has few adjustment parameters, and the model structure is reflected by the support vector points that can provide the most information characteristics.

Greatly reduce the complexity of modeling. Support vector machine is currently the best method in fault monitoring, diagnosis, and prediction. Support vector machine adopts a new learning mechanism, introduces kernel function, realizes the principle of structural risk minimization, and has the boundary of minimizing empirical risk and VC dimension. The actual risk is relatively small. Therefore, the support vector machine-based method has a strong generalization ability, and it can still guarantee a fairly high accuracy even in a long interval prediction.

4) Information fusion and other methods

Information fusion is a process of comprehensively processing multi-source information at multiple levels, where each level reflects a different level of abstraction of the original data [12]. The purpose of information fusion in EHM is to improve engine fault diagnosis, performance. 
Can predict and estimate the remaining life of key components and other aspects of accuracy and credibility; the ultimate goal is to maximize the extraction of useful information from different data through information fusion, so as to obtain a complete

Face diagnosis and prediction, and finally make the best decision. The F117 engine jointly developed by Dryden Flight Research Center and PW Company uses the current mature EHM information fusion system [13], as shown in Figure 5. The system adopts a component-based, open, and hierarchical architecture, which can complete signal processing, data analysis, health assessment, fault diagnosis reasoning, and maintenance activity recommendations. EHM's signal analysis methods also include parameter sensitivity analysis, model estimation, fuzzy logic, genetic algorithm, Bayesian theory, expert system and decision tree methods.

\subsubsection{Fault Diagnosis and Prediction Technology}

A typical fault diagnosis and prediction process include data collection, processing, transmission, feature extraction, fusion, status monitoring, fault diagnosis, and support decision-making, as shown in Figure 6. Fault diagnosis and prediction is the highest level of reasoning in the EHM system. Essentially, it can be divided into three types of methods based on model, knowledge and data [14].

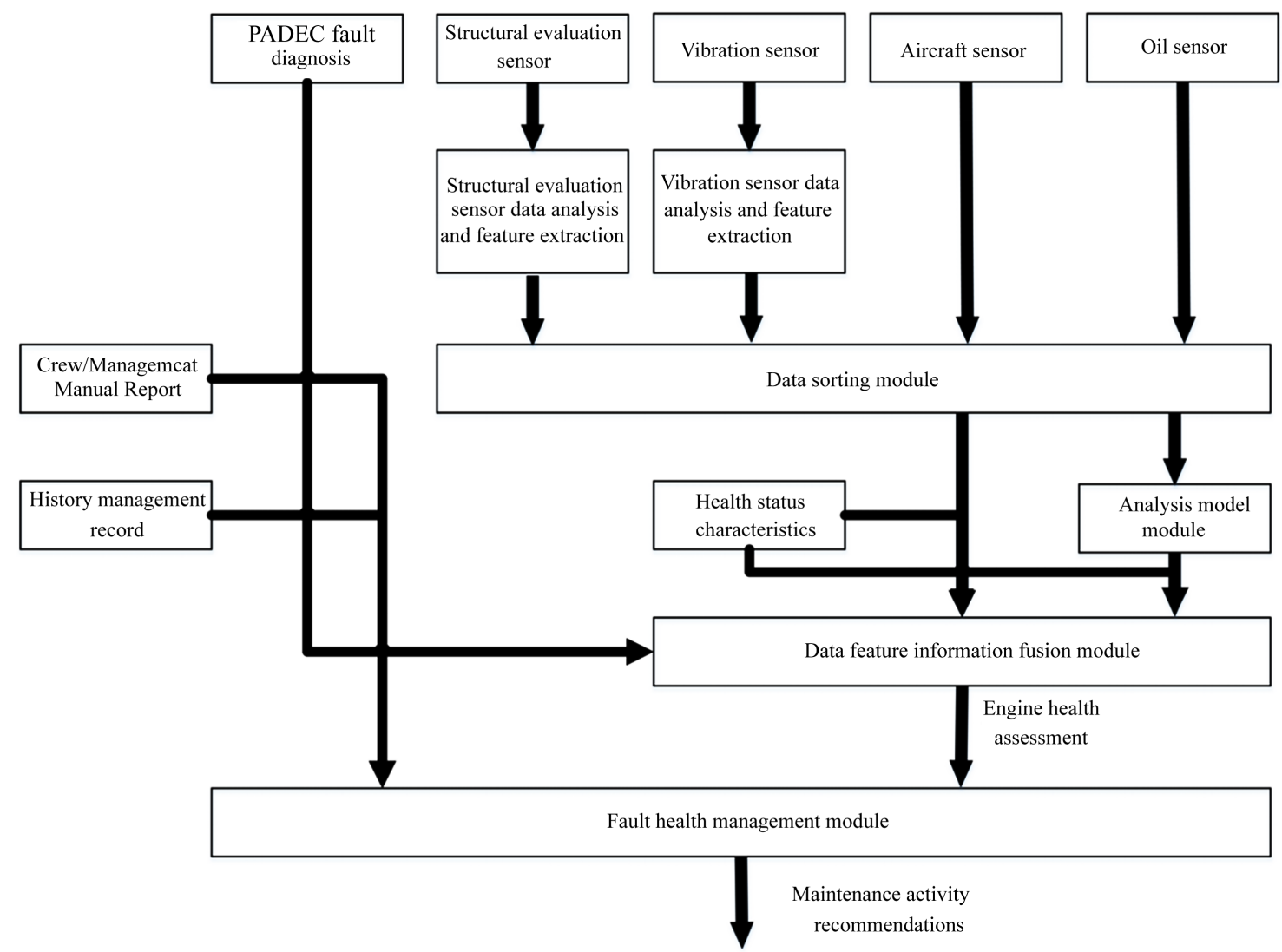

Figure 5. EHM information fusion architecture. 


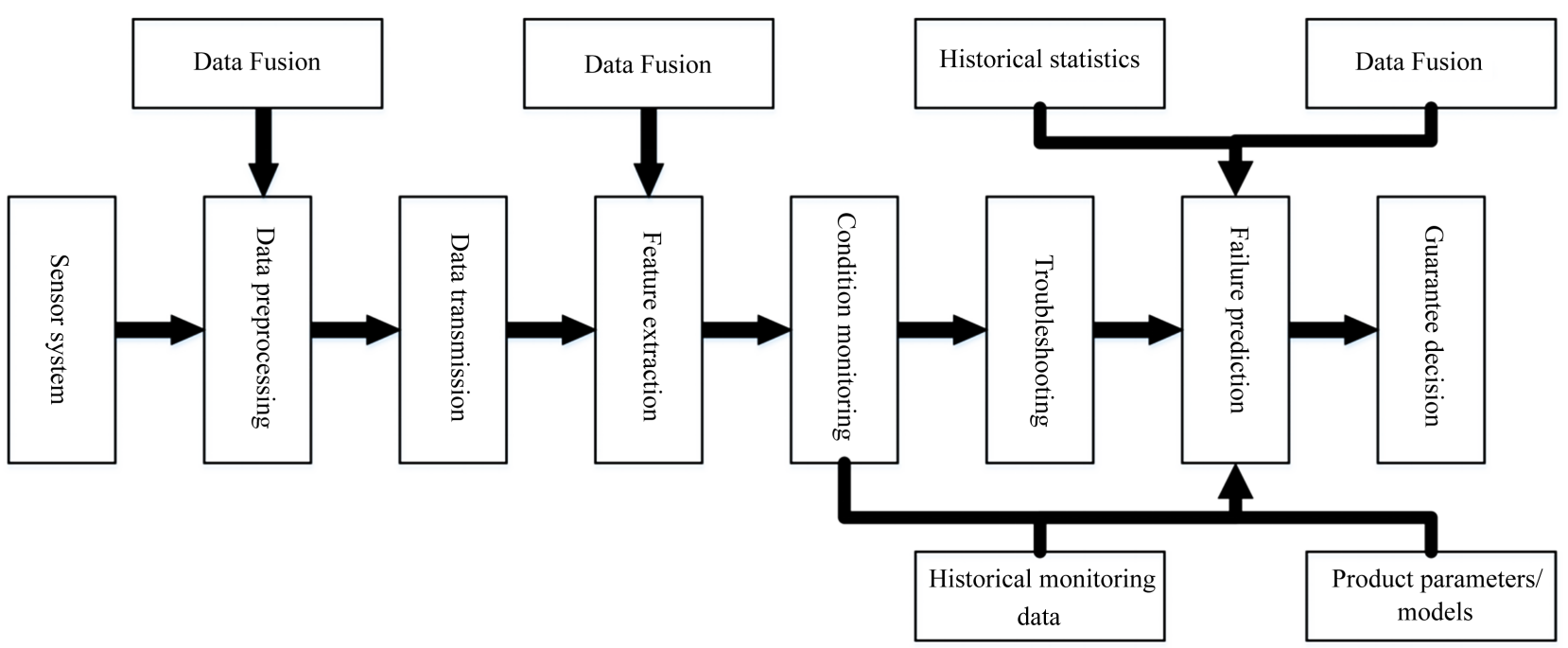

Figure 6. Fault diagnosis and prediction process.

Model-based fault diagnosis and prediction technology must have engine mathematical models and component failure models. The biggest advantage is that it can meet real-time performance, so this technology is often used in airborne EHM systems. Since the engine is a complex system, it is difficult to determine its model, so the development of this technology is subject to certain restrictions. Knowledge-based fault diagnosis and prediction technology are realized based on the expert knowledge and experience of various disciplines of the engine. At present, the most commonly used methods are expert systems and fuzzy logic. Expert systems have difficulties in acquiring knowledge, and their development has been limited; fuzzy logic is still in the research stage, and some problems need to be solved urgently.

The biggest advantage of data-based fault diagnosis and prediction technology is that it does not require precise engine mathematical or physical models. The most typical representative is neural network. For complex systems such as engines, due to the difficulty of diagnosis and prediction research, it is difficult to use a single method to ensure its application effects. The use of hybrid methods to integrate their respective advantages and further improve the comprehensive performance of fault diagnosis and prediction is an inevitable trend in the development of fault diagnosis and prediction technology. The engine data-driven model based on the fusion of the three methods is shown in Figure 7.

\subsection{Integration Technology}

The EHM system is a complex information system that can integrate its own different modules in accordance with certain rules and requirements. It not only improves the open efficiency of the EHM system, realizes the sharing of the capabilities of each module, but also helps to weigh and analyze the system to meet different functional requirements. Different configurations or architectures provide a set of options and solutions for the EHM system to meet different needs. 


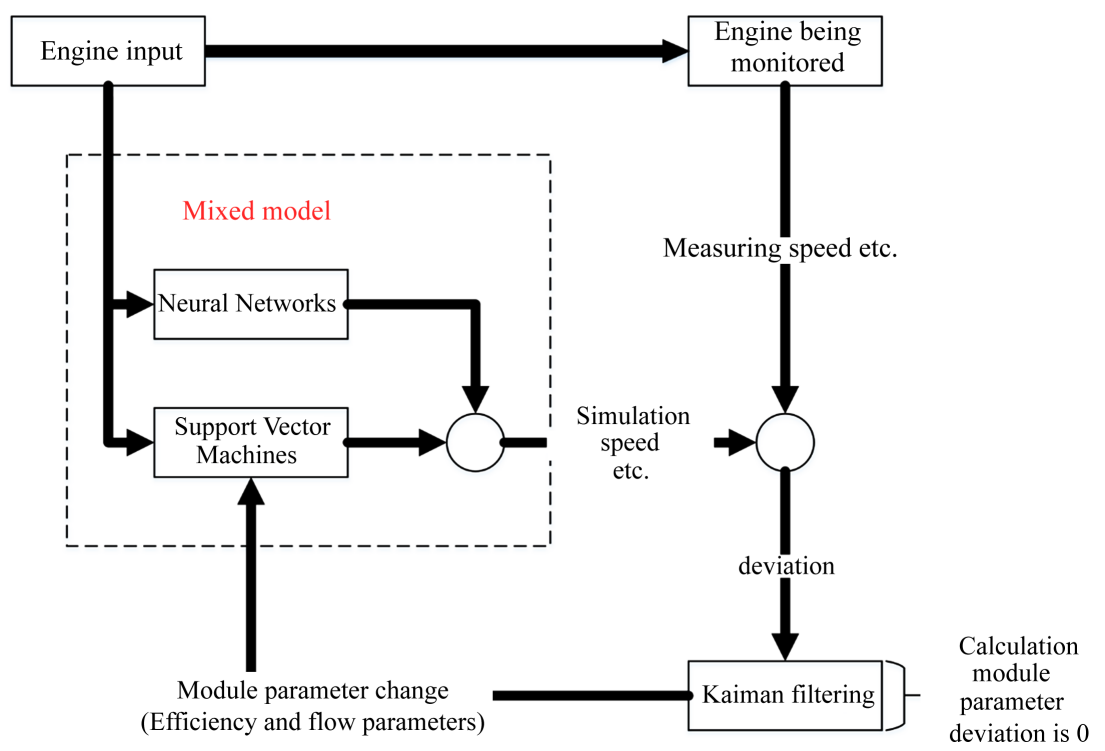

Figure 7. Engine data-driven model.

In addition, the comprehensive integration of EHM system and engine control system (especially FADEC system) is an important development trend for new generation engines. In addition, other airborne systems, such as cockpit display system, flight management system, mission management system, etc. are connected with multiple links in the EHM system. The use of digital systems and data buses can enable the EHM system and other systems to achieve an integrated design, thereby improving the open efficiency of each system, reducing costs, and reducing the space and quality occupied.

\subsection{Communication Technology}

Communication technology enables the engine onboard and ground subsystems and crew maintenance personnel to contact and communicate with each other, so that EHM data can achieve the best control in the engine application in the FADEC system, thereby assisting the navigation system to communicate warnings, warnings or suggestions. And it can help the navigation system to make decisions under abnormal conditions, or help agile decision-making communication to make endurance plans. Communication technology directly affects the implementation of EHM system functions, and communication modules are also an important part of EHM system design.

\section{Design Requirements of EHM System}

EHM system design technology has two performance indicators [14]: safety and life cycle cost. These two indicators are usually in inverse proportion. The high security of the EHM system comes at the expense of the expensive cost of its hardware, software and infrastructure. Therefore, the design of the EHM system must fully consider various factors to achieve the optimal configuration of these two indicators. 


\subsection{Security Design}

Safety design is the rational application of health management technology to solve engine safety issues, so that engine operation and maintenance have more high security. When designing the EHM system, make full use of advanced sensor technology, communication technology and monitoring, forecasting and condition management technology to ensure the safety, reliability, and low cost of the engine, and also satisfy buyers, engine manufacturers, sellers and engineering Requirements of field personnel.

\subsection{Life Cycle Cost Design}

In the design of the EHM system, there are many methods that can significantly reduce the cycle cost of the engine life and the related costs of operation and maintenance. First, manufacturers can use EHM technology to reduce engine production costs and initial costs; second, users can use the performance of the EHM system to reduce operating costs and maintenance costs; third, costs can be reduced by quickly operating suppliers. In the current cost-conscious aviation environment, the company provides a fast engine management plan (FMP), allowing operators to establish a fixed cost through a contract for maintenance and transfer of risks to FMP suppliers; in addition, EHM can also be used Integration and the use of fault monitoring, diagnosis, prediction and communication to reduce production costs and maintenance costs.

\subsection{Software Development}

System software development adopts modular programming ideas, and adopts a variety of software development tools according to the functional requirements of different modules, and system integration of functional modules. For example, the expert system is developed with CLIPS (CLanguage Integrated Production System) and embedded in the main program in the form of a dynamic link library. The database platform can choose MicrosoftSQL Sever 000 enterprise edition, and realize the connection with the main program through the database connection toolkit of Lab Windows.

\section{Summary}

1) In order to design a complete, safe, stable and reliable EHM system, we must first accelerate the development of advanced hardware products and develop high-precision, integrated, adaptable to harsh environments, strong reliability and high-speed new sensors to measure parameters that cannot currently be measured and play a key role in comprehensively assessing the health of the engine; the development can adapt to harsh environments and large-capacity high-speed storage equipment and communication equipment to meet the real-time needs of EHM; it is necessary to optimize the configuration and rational use of each hardware to achieve the best overall performance of the entire hardware [13]; secondly, it is necessary to fully grasp the mechanical perfor- 
mance data of various aspects of the material, and establish an accurate prediction model to predict the development trend of failure; third, further research and improve advanced information fusion and intelligent data fusion technologies; finally, integrate EHM into the engine design to improve the monitorability, diagnosability, predictability and mitigation of the engine.

2) The development of the current EHM system design technology is embodied in the system-level integrated application as the traction to improve the accuracy of fault diagnosis and prediction, expand the scope of application objects of health monitoring, and support the development based on condition maintenance and independent guarantee. In short, EHM is developing towards real-time, intelligent, integrated and networked directions.

\section{Conflicts of Interest}

The author declares no conflicts of interest regarding the publication of this paper.

\section{References}

[1] Long, B., Sun, Z.M. and Jiang, X.W. (2003) Research on Spacecraft Integrated Health Management System. Aerospace Control, No. 2, 56-61.

[2] Li, A.J., Zhang, W.G. and Tan, J. (2007) Overview of Aircraft Health Management. Electro-Optics and Control, 14, 79-83.

[3] Clark, G.J., Vian, J.L., West, M.E., et al. (2007) Multi-Platform Airplane Health Management. IEEE Aerospace Conference, Big Sky, MT, 3-10 March 2007, 1-13. https://doi.org/10.1109/AERO.2007.352944

[4] Keller, K., Wiegand, D., Swearingen, K., et al. (2001) An Arxhitecture to Implement Integrated Vehicle Health Management System. IEEE Systems Readiness Technology Conference, Valley Forge, PA, 20-23 August 2001, 1080-7225.

[5] Zeng, S.K., Pect, M.G. and Wu, J. (2005) Current Status and Development of Fault Prediction and Health Management (PHM) Technology. Acta Aeronautica Sinica, 26, 626-632.

[6] Kobayashi, T. and Simon, D.L. (2007) Integration of On-Line and Off-Line Diagnorithms for Aircraft Engine Health Management. ASME Turbo Expo 2007: Power for Land, Sea, and Air, Montreal, Canada, 14-17 May 2007, 649-659. https://doi.org/10.1115/GT2007-27518

[7] John, R., et al. (2001) Incorporating Residual Stresse in Life Prediction of Turbine Engine Disks. NATOAV Symposium on Age Mechanism and Control, Manchester, UK, 1-15.

[8] Wang, P. (2007) Advanced Sensing Technology of JSF Engine Health Management System. Aviation Power News, 1000-8829(2007)03-0027-03.

[9] Roemer, M.J., Tang, L., Kacprzynski, G., et al. (2006) Simulation-Based Health and Contingency Management. 2006 IEEE Aerospace Conference, Big Sky, MT, USA, 4-11 March 2006, 1095-323X.

[10] Tu, F., Ghosal, S., Luo, J., et al. (2007) PHM Integration with Maintenance and Inventory Management Systems. 2007 IEEE Aerospace Conference, Big Sky, MT, 3-10 March 2007, 1-12. https://doi.org/10.1109/AERO.2007.352918

[11] Wang, S. (2007) Research on Computer Aided Equipment Planning Support Work 
Platform. Beijing University of Aeronautics and Astronautics, Beijing.

[12] Tay, F.E.H. and Cao, L.J. (2001) Applications of Support Vector in Financial Porecasting. Omega, 9, 309-317. https://doi.org/10.1016/S0305-0483(01)00026-3

[13] Volponi, A.J., Brotherton, T. and Luppold, R. (2004) Development of an Information Fusion System for Engine Diagnostics and Health Management. AIAA $1^{\text {st }}$ Intelligent Systems Technical Conference, Chicago, 20-22 September 2004, 6461. https://doi.org/10.2514/6.2004-6461

[14] Wang, S., Wang, R.Q., Chen, Z.Y., et al. (2009) Overview of Aeroengine Health Management. Gas Turbine Experiment and Research, 1, 51-58.

https://doi.org/10.2166/aqua.2009.085 